\title{
Considérations sur les tendances actuelles d'établissement des conduites forcées de grande puissance sous haute chute
}

\author{
Present design trends for high-output penstocks \\ operating under hig head
}

\author{
PAR G. FERRAND \\ PHÉSIDENT D'HONNTUR ET INGÉNIEUR-CONSEIL OOE LA SOCIÉTÉ D'ENTREPRISE DE MONTAGES (s.D.E.M.)
}

Dans les décennies qui ont précédé l'année 1949, l'évolution de la technique en matière de conduites forcées, notamment pour les hautes chutes à grande puissance, a été particulièrement importante.

De 19:9 d 1956 , l'évolution a été moins speclaculaire; on a cependant bu natitre de trèss puissants aménagements et la mise en cuvve de tuyaux auto-frettés surpressés à Pragnères. Depuis 1956 , des aménagements plus importants que les précédents, tels que Roselend par exem,ple, voient le jour.

En face de ces réalisations et des projets $\dot{a}$ l'étude, le maître de l'œuvre a été amené à revoir les règles des cahiers des charges existants, notamment sons l'angle de la sécurité d'exploitation.

De nouvelles méthodes de calcul, basées sur l'emploi des mathématiques les plus modernes, permettent en effet de déterminer les coefficients de sécurité réels sous lesquels sont exploitées, en toute sécurité, les nombreuses et importantes conduites forcées que nous avons étudiées et installées. Partant de ces précédents, ces nouvelles méthodes permettent de fixer, pour les nouveaux aménagements, des coefficients de sécurité optimums, bien appropriés à leurs conditions d'installation et d'exploitation, sur des bases indiscutables.

En dehors de ces tendances relatives ì la sécurité, d'autres se manifestent qui touchent à l'amélioration de la qualité des matières utilisées, par de plus grandes exigences dans leur élaboration et leur contrôle chez le fonrnisseur, mais aussi par un contrôle plus rigoureux de la fabrication, grâce $\dot{a}$ des moyens modernes, parmi lesquels on peut citer les ultra-sons, dont l'utilisation est maintenant bien au point.

Les tendances actuelles résident aussi dans la fixation d'un maximum pour la valeur du rapport $\mathbf{E} / \mathbf{R}$ de la limite élastique à la résistance et d'une éprenve en usine des éléments d'une conduite forcée, faite $\dot{a}$ une pression telle que In contrainte correspondanle soit nettement inférieure à $\mathrm{E}$. Cette disposition priverait le
Considerable progress in penslock design was made during the last few decades leading up to the yerr 1949, especially in connection with high-output plant operating under substanlial heads.

Though the rate of progress between 1949 and 1956 was less spectacular some very important high-output power projects were nevertheless undertaken during this period, which also saw the commissioning of the prestrained "autofretted" penstocks at Pragnères.

Even more ambitious projects are now being undertaken, such as the Roselend dam for instance.

The development of these and future verly largescale schemes has made it necessary to revise certain specifications, especially thase in connection with operational safety.

New design methods have been developed, which are based on the most recent mathematical methods; these have been used to determine the true operational safety factors for the man! large penstocks the Author has designed and installed. The new methods enable optimum safety factors to be determined for new projects, which are not only matched to the installation and operational conditions in each case but are also based on thoroughly sound data.

In addition to safety, progress has also been made in the quatity of the materials used by raising the manufacturing and inspection standards, the latter both at the raw material processing and menufacturing stages. This improvement has been made possible by the modern technical equipment now available for the purpose, especially ultra-sonic equipment, which has been developed to a high degree of reliability.

The present procedure is to set a maximumvalue for the elastic limit/strenght ratio $E / R$ and to factory-test the penstock sections at a pressure resulting in a stress much lower than $E$. This method alone cannot guarantee a true and tangible safety factor of at least 2 
maitre de l'aubre de la garantie d'un coefficient de sécurilé rél et langible au moins égal a 2 , si elle n'était accompagnée de la fixation d'un coefficient de sécurité tel que la «régle d'or» d'ćpreuve à $2 \mathrm{P}$ soit neanmoins conservée.

Les tendances nouvelles touchent aussi a la prow tection absolue contre les éléments extérieurs quels qu'ils soient. On tend aussi d admettre. enfin que la fourniture d'une conduite forcie, et les trawaux de génie civil qui s'y ratiachent, sont des éléments inséparables l'un de l'autre dans le cadre de l'économie générale. L'importance du génic civil étant fonction directe de la structure de la conduife, ce n'est pas toujours la solution où la conduite est la moins coûteuse $q u i$, en défnitive, est la plus avantagense pour le maître de l'ouvre.

On tend enfin à uliliser, en tuyaux soudés, des aciers $a$ tres haute limite élastique obtcnue par trempe et revenu, dont l'emploi est particulièrement intéressant à envisager en tuyaux anto-frettés d double paroi lorsque les frettes sont trop couteuses du fait de lents dimensions.

En 1949, dans un article \& Les Tendances nouvelles »(1) et, plus récemment, dans un autre: « L'allégement des conduites forcées modernes $\$(2)$, nous marquions l'évolution de la technique d'établissement des conduites forcées, notamment pour les hautes chutes de grande puissance, d'où il ressortait que les économies de poids réalisées furent sensationnelles, grâce en particulier à la mise en cuvre d'aciers à haute et très haute limite élastique.

Cinquante années d'expérience nous araient aussi amenés à envisager la réalisation de nouvelles économies, en réduisant la valeur $2,5 \mathrm{du}$ coefficient de sécurité stipulée au cahier des charges de la Société Hydrotechnique de France, à la condition expresse de respecter la « règle d'or » d'épreuve à $2 \mathrm{P}$ faite en usine $(\mathrm{P}=$ pression de calcul) de chaque élement de conduite forcée. Dans le but de donner au maître de l'reuvre une sécurité d'exploitation totale, nous allions même encore plus loin en préconisant que cette épreuve devrait être poussée jusqu'à $c P(c=$ coefficient de sécurité par rapport à $\sigma_{0,2}$ ), les tuyaux construits étant systématiquement surpressés.

On trouvera les raisons de ce point de vue dans un article : «A propos d'un accident survenu à une conduite forcée après quarante ans d'exploitation $\gg(3)$.

Celles-ci peuvent se traduire par cette définition :

Une conduite forcée construite en tuvaux surpressés (simples ou auto-frettés) est

(1) La Howille Blanche, n" 3, 1949.

(2) Annales des Ponts el Chatssées, no 2, mars-avril 1956.

(3) La Houlle Blanche, $n^{\circ} 1$, janvier-février 1953. unless a safety factor is also assumed such that the "golden testing rule" of $2 P$ is respected. other new trends are: 1) to aim at providing all ontside components with an absolutely reliable protection and 2) to consider the penstock and the civil engineering works with which it is associated as a single unit from the general economic point of view. Since the anount of civil engineering work depends directly on the structure of the penstock, low penstock costs do not necessarily also result in cheap overall layouts.

Hert-treated and tempered steels with a verl high elastic limit are becoming increasingly popular for welded penstock construction. These are of particular interest for donble-wall "autofretted" penstocks when ordinary hooping would be prohibitely expensive in view of its large dimensions.

pratiquement à l'abri de risques de ruy'lure, tant qu'une surpression provenanl. d'une circonstance «accidentelle exceptionnelle » ne détermine pas dans la conduite des pressions momentanées amenanl dans la tôle ou dans la frette des contraintes proches de la limite de rupture des aciers les constituant.

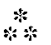

Dès 1949, alors que l'on utilisait largement et depuis longtemps les tuyaux auto-frettés et les tuyaux surpressés, selon la formule dite « conduite unique », nous pensions à l'utilisation des tuyaux surpressés auto-frettés et anto-frettés surpressés sur lesquels avaient été faits des études et essais très poussés sur prototypes. Mais ces procédés ne furent appliqués pour la première fois à l'échelle industrielle qu'en 1952, à Pragnères (4).

Jusqu'en 1956, il n'y eut pas d'évolution spectaculaire dans la technique et la technologie des conduites forcées. Ce sont plutôt des réalisations très puissantes qui naquirent. Depuis, une certaine évolution se manifeste qui, sans être aussi frappante que lors des années précédentes, comporte des tendances notables touchant davantage à la sécurité qu'à l'économie.

Le cahier des charges de la S.H.F. était alors le

(4) « Les conduites forcées de Pragnères 》. Edition actobre 1955 des Ets Bunchayer et Viallet al de Ia Sociate Dauphinoise d'Etudes et de Montages.

(5) La Honille Blanche, $n^{\circ} 1$, janvier-lêvrier 1960 , * Aménagement hydróleclrique de Roselend. - Conduite forcée de l'usine de La Bathie $»$, par R. Canturer. 
seul, pour la France, à définir les règles de l'art en matière de construction de conduites forcées métalliques. Depuis, l'Electricilé de France élabore son propre cahier des charges, différant sur certains points de celui de la S.H.F., le complétant sur d'autres.

La mise en cuvre de l'aménagement de Roselend (5), actuellement en voie de terminaison, et les projets plus importants encore qu'elle étudie, dont la réalisation est prochaine, ont amenć l'E.D.F. à revoir les règles établies. Par rapport aux précédents, tels Malgovert et Pragnères par exemple, Roselend comporte en effet une extrapolation importante el des projets en étude en constituent une autre par rapport à Roselend.

A l'étranger, où des aménagements du mème ordre sont en cours ou en projet, les objectifs du maitre de l'œuvre sont les mêmes que ceux de l'E.D.F. C'est ainsi qu'un cahier des charges «européen , rejoignant sur de nombreux points celui de l'E.D.F., est en voie d'élaboration.

\section{央:}

Les définitions el les règles de ces cahiers des charges tendent à fixer des coefficients de sécurité sur des bases indiscutables dénuées d'empirisme. De nouvelles méthodes de calcul s'appuyant sur les mathématiques les plus modernes, qui sont la transposition, dans le domaine des conduites forcées, de I'élastieité appliquée - le support mathématique utilisé étant actuellement demeuré classique (calcul matriciel par exemple) - permettent en effet de le faire avec la rigueur nécessaire.

Ces nouvelles méthodes facilitent en effet. Ia recherche du coefficient de sécurité optimal, en domnant à l'ingénieur - avec les moyens dont il dispose maintenant pour chiffrer très rapidement les formules découlant d'équations les plus compliquées - la possibilité de déterminer avec précision les tenseurs des contraintes.

Elles permettent ainsi de déterminer les coeffcients de sćcurité réels sous lesquels fonctionnent les conduites forcées exislantes et, compte tenu des résultats obtenus, de fixer, pour les conduites nouvelles et fulures, des coefficients de sécuritó bien appropriés à leurs conditions d'aménagement et de fonctionnement (situation géographique, importance, pérennité, structure, mode d'installation, caractère des efforts).

Les conduites forcées existantes que nous avons étudićes jusqu'à ces dernières années l'ont ité en partant de considérations parfoìs empiriques, mais valables en raison d'une très longue expérience qui, remarquons-le en passant, permet à l'ingénieur de baser ses calculs sur des hypothèses sûres. L'exactitude de ceux-là dépend en effet de la valabilité de celles-ci.

Par ailleurs, les calculs étaient faits dans le cadre du cahier des charges de la S.H.F. définissant un coefficient de sécurité « $c 》$ tel que sous $c \mathrm{P}$ les contraintes soient au plus égales à $\sigma_{0.2}$ et pour un état de contraintes unidimensionnelles. Il est vrai que pour les conduites importantes et notamment pour les conduites auto-frettées à paroi mince, nous vérifions a posteriori la valeur des contraintes bidimensionnelles, selon la formule (6) dont l'application nous avait été recommandée en 1932 lors d'essais faits en compétition internationale au Laboratoire Fédéral de Zurich (7) sous sa direction, par l'éminent professeur Ros - formule qui n'est autre que celle de Hencky von Mises ramenće à la forme hidimensionnelle - pour laquelle il nous avait également recommandé que la valeur sous pression de marche $\mathrm{P}$ de la contrainte fictive trouvée ne soil pas supcrieure à $2 / 3\left(\sigma_{0,2}\right)$.

Ces conduites existantes, dont lous les élẻments ont été éprouvés en usine à $2 \mathrm{P}$ au moins et dont l'épreuve générale après montage, dont nous avons souvent souligné l'importance, a èté faite conformément aux prescriptions du cahier des charges S.H.F., ont reçu la sanction de l'expérience et fonctionnent cn toule sécurité. Rappelons en passant que l'épreuve après montage est souvent plus dure que l'épreuve en usine, notamment pour les tuyaux de la partie supérieure d'une conduite qui sont les plus sensibles aux surpressions provenant de coupures de débit de forme instantanée.

L'établissement de ces conduites est donc correct et les vérifications déjà faites sur les plus importantes d'entre elles en sont la confirmation aussi bien pour les culottes de distribution sphériques type G.F. que pour les différents types de tuyaux généralement utilisés.

Il se peut cependant que les calculs eftectués fassent ressortir entre conduites ou même entre tuyaux d'une même conduite des différences plus ou moins grandes entre les valeurs des contraintes équivalentes. Cela dépend beaucoup de la valeur du rapport $e / \mathrm{D}$ de l'épaisseur au diamètre, qui conditionne la plus ou moins grande flexibilité d'un tuyau.

En effet, quel que soit $e / D$, les calculs sont faits sur les mêmes bases. Or, les tuyaux souples ne se comportent pas comme des tuyaux raides, c'est-à-dire en flexions latérales comme des anneaux rigides, et en flexions longitudinales

(6) $\sqrt{\mathrm{T}^{2}+\left(\mathrm{T}_{r}+\mathrm{T}_{1}\right)^{2}-\mathrm{T}\left(\mathrm{T}_{1}+\mathrm{T}_{1}\right)}$ ò $\mathrm{T}=$ contrainte tangentielle dans la paroi; Ty contrainte de flexion de la paroi entre frettes: $T_{2}$ contrainte longitudinale; $T$, ef ' $T_{i}$ pris avee leurs signes.

(7)) Tuyaux auto-frettés pour conduites forceses. ...Frettage par extension de la paroi a froid. - SDEM, dition 1934. 


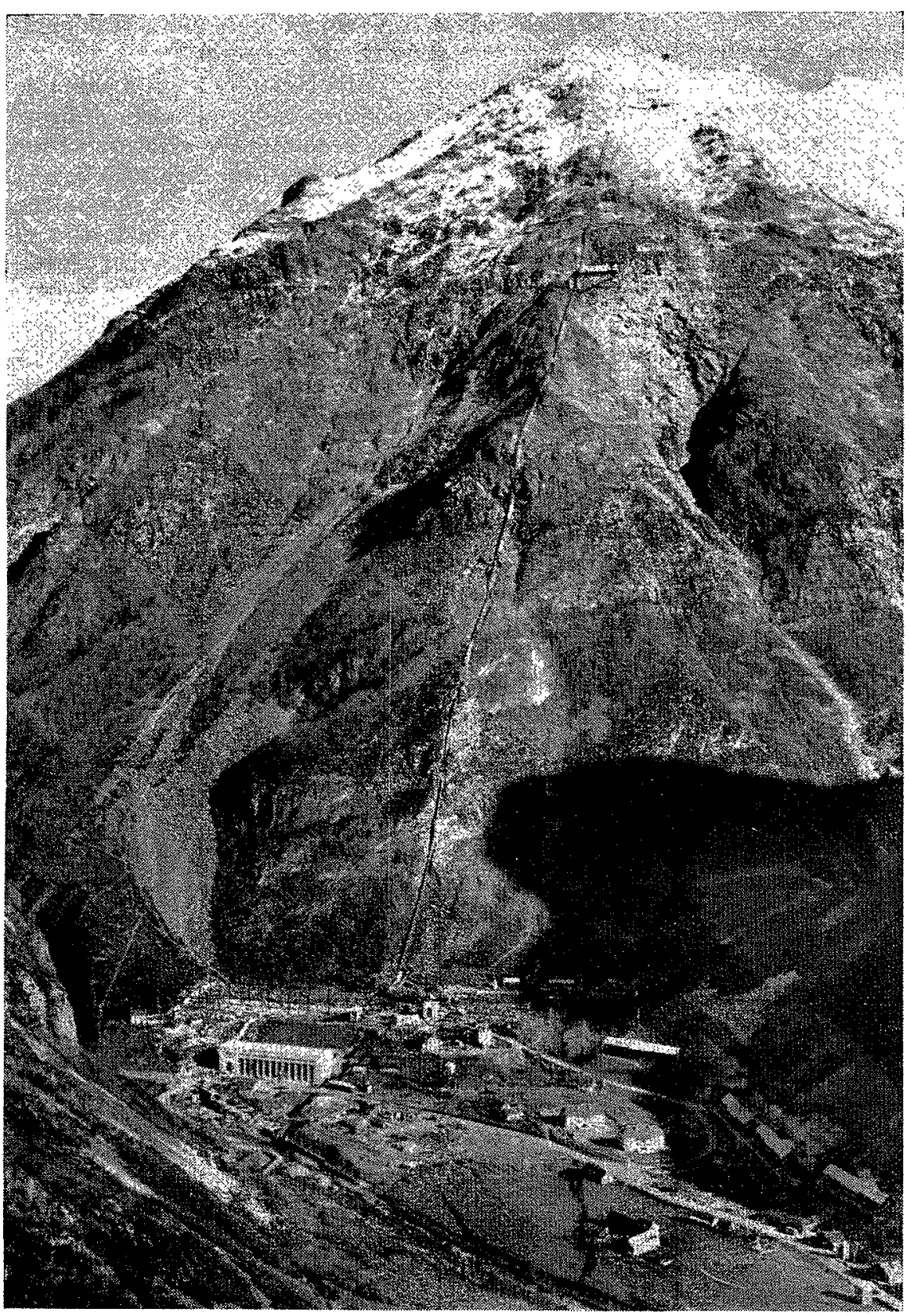

Fic. 1

Vue d'ensemble de la conduite forcée de Cap de Long, chute de Pragnères.

(Photo Alit, Bagnires-de-Bigorre.) comme des poutres rigides, soit qu'il s'agisse de très grandes portées entre les appuis d'un troncon de conduite, soit qu'il s'agisse de la paroi entre les frettes d'un tuyau auto-fretté. Rappelons à ce sujet le cas de la conduite soudée d'Izourt (8) et celui de la conduite auto-frettée de Pralognan (9) qui se sont trouvées, accidentellement et en plein service normal, suspendues comme des câbles avec des portées de 80 à 100 fois leurs diamètres, sans donner lieu à des déformations permanentes latérales sur les appuis d'extrémité ou longitudinales entre ces appuis.

(8) Conduites forcées. - Technique 1941, EBV/SDEM.

(9) La Houille Blanche, $\mathrm{n}^{\circ}$ 1, janvicr-février 1953.
Citons aussi l'exemple, donné par Aragon (10), pour le calcul d'une tôle de bordé de portes d'écluses où les bandes sont assimilées à un câble à flexion parabolique.

Citons enfin le cas de la vieille conduite rivée de Saint-Jean-de-Maurienne, de $3,300 \mathrm{~m}$ de diamètre, reposant sur des berceaux en maconnerie à $90^{\circ}$, espacés de 6 mètres et dont l'épaisseur minimale au sommet est de $8 \mathrm{~mm}$. Cette conduite fonctionne en service continu depuis plus de 50 ans sans accroc - mis à part le sabotage par "xplosif dont elle fut victime, à la fin de la der-

(10) Ernest Aragon : « Rósistance des matériaux», tome III. 
nière guerre mondiale - et sans que l'on ait constaté de déformations permanentes sur les appuis.

C'est la formule de Hencky von Mises, que l'on utilise maintenant le plus généralenent, qui fixe, en fonction des contraintes agissant simultanément, la valeur de la contrainte équivalente. Elle est donnée pour un état de contrainles tridimensionnelles, mais en matière de conduites forcées, les contraintes radiales et de cisaillement sont négligeables, l'état de contrainte devient un état plan caractérisé par les contraintes circonférentielle et longitudinale. La contrainte équivalente est alors déterminée pour des efforts bidimensionnels.

On ne peut dissocier les calculs des coefficients de sécurité de ceux des contraintes, mais au contraire, plus on veut préciser ce que l'on entend par sécurité, plus on est amené à faire des calculs précis.

Dans le développement de probabilités conposées, il reste la notion même de probabilité des causes.

Dans le cas des conduites forcées, c'est évidemment la probabilité de surpression qui devient le facteur déterminant.

On connaît avec une assez bonne precision la valeur maximale de la surpression qui peut survenir en marche normale et sa répartition le lons din profil d'une conduite déterminée.

On connaît aussi l'ordre de grandeur de la surpression maximale accidentelle qui peut survenir à la suite d'accidents touchant aux organes de distribution aux turbines, pouvant donner lieu à des coupures instantanées de débits, d'autant plus importante que le nombre d'organes de dis-
FiG. 2

Vue d'ensemble de la conduite forcée du Pouget.

Hauteur de chute brute : $\quad 460 \mathrm{~m}$ Débit : $30 \mathrm{~m}^{3 / \mathrm{s}}$ Puissance: $160000 \mathrm{ch}$ Diamètres : $\quad 2,500 / 2,700$ /a (Photo S.D.E.M., Grenoble.)

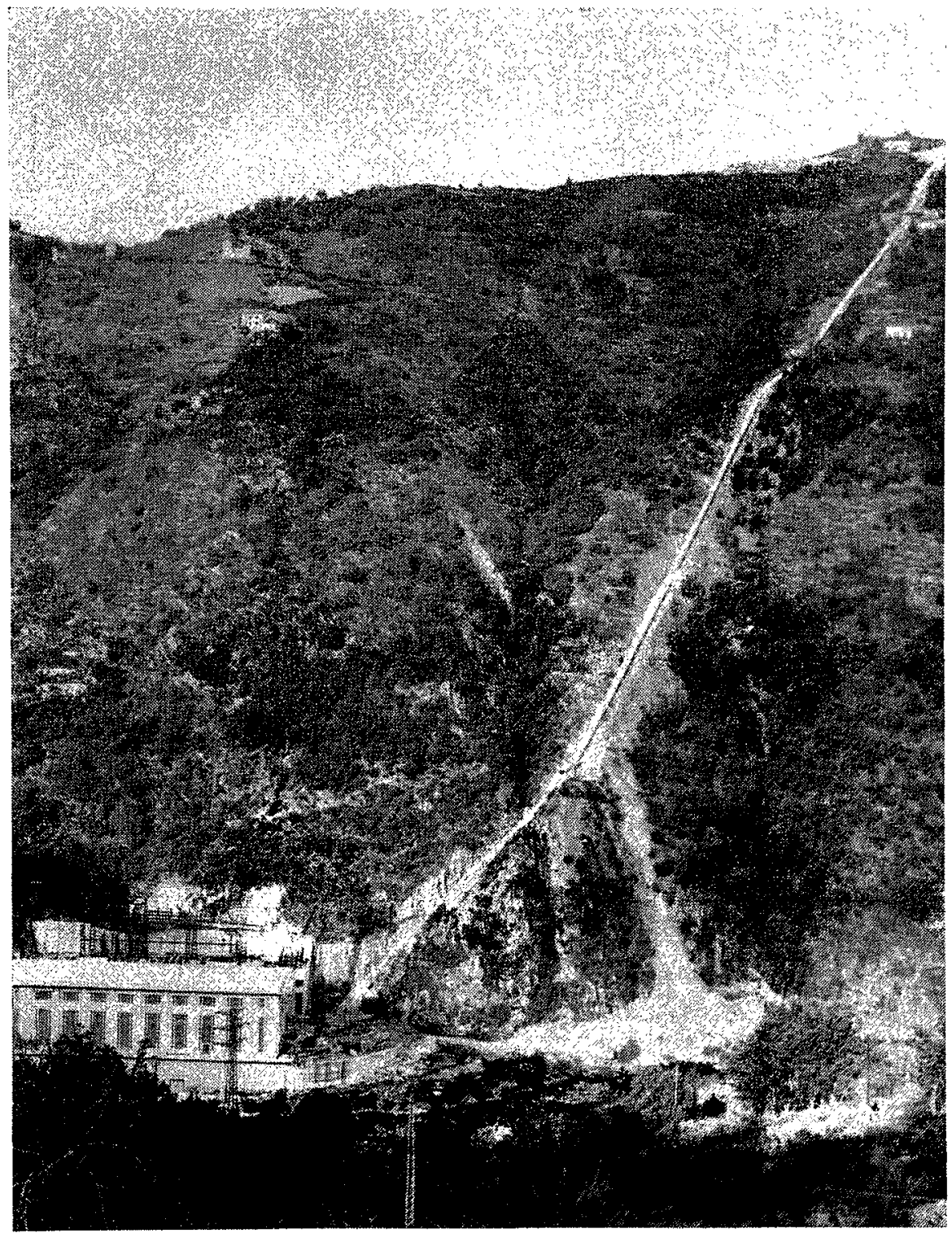


Iribution est plus réduit. La répartition de celte surpression le long de la conduite est également comnue. Mais il est d'autres causes de surpression cque l'on ne peut connaître et dont, par conséquent, il est impossible de tenir compte avec précision.

Cependant nous pouvons dire qu'une très longue expérience nous a permis d'enregistrer des surpressions «accidentelles exceptionnelles »-qui ne peuvent se produire que tres rarement et peut-être jamais dans la vie d'une conduite forcée - atteignant la valeur mème de la pression maximale normale de marche. C'est d'ailleurs pourquoi il y a lieu d'attacher tant d'importance à l'essai, fait en usine à $2 \mathrm{P}$, de chaque élément de conduite.

Des commentaires sur « I'interdépendance des calculs des contraintes et de la sécurité $\gg$. se rapportant à la conduite forcée de Kurobe IV (Japon), donl les caracléristiques générales sont:
Hauleur de chule brute. .

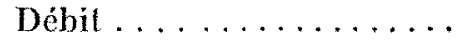

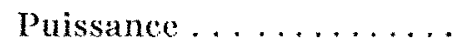

. . Diamètre . . . . . . . . . . .

-... Pression maximale de mus-

che au collecteur....... définissent la notion de sécurité en montranl en même temps l'avantage écrasant des tuyaux autofrettés en sécurité d'exploitation.

L'intérêt qui s'attache à ces commentaires qui ont amené le maître de l'œuvre à donner la préférence à la solution autofrettée (11) - nous amène à les reproduire ici dans leur texte intégral.

Les voici :

\section{1) Généralités.}

On ne peut pas dissocier la notion du coefficient de sécurité des méthodes mises en ouvre pour déterminer les contraintes.

Pourtant, le coefficient de sécurité lui-même est une notion relativement arbitraire. La conception la plus classique repose sur une expérience établie. On sail par exemple que, dans les calculs de pont ou d'avion, en adoptant tel coefficient, on ne prend pas de risques tout en réalisant une économie saine.

Mais, même dans un domaine aussi exploré, on a assisté à une baisse des coefficients de sécurité, au fur et à mesure de la connaissance des propriétés mécaniques des matériaux et que les méthodes de calcul de contraintes se précisaient.

(11) Présenté par la Sté Franco-Italienne \& COFOR 》 constituée par les sociétés francaises EBV/SDEN et par la société italienne TERNI.
Il est une autre voie touchant la philosophie sur laquelle on peut essayer de s'engager : c'est celle de la notion probabilistique. En effet, un matériau est défini par des clauses de réception qui traduisent des caractéristiques minimales, une structure est définie par des cotes qui traduisent des dimensions théoriques, les efiorts représentent une combinaison de causes de probabilité diverses : bref, on arrive à dire : pendant la vie de tel ouvrage, on a une probabilité de $10^{-n}$ que les contraintes en une partie de la structure deviennent une cause de destruction.

Et, fait remarquable, en suivant cette voie, alors qu'on manie des valeurs qui sont aléatoires, on est conduit à déterminer, avec le plus d'exactitude possible, les tenseur's de contraintes afférents à chaque cause.

Donc, de toute façon, non seulement on ne. peut pas dissocier les coefficients de sécurité des calculs de contraintes, mais au contraire, plus on veut préciser ce que l’on entend par sécurité, plus on est amenó à faire des calculs précis, mettant en ouvre les mathématiques les plus modernes.

Et, par un enchainemenl curieux, qui est l'ailleurs celui du progres scientifique, mieux on sait calculer, plus on définit de critères cumulatil's dont l'ensemble forme la sćcurité.

Pour revenir au sujet précis des conduiles forcées, l'avantage des conduites auto-frettées est. écrasant, que l'on se place au point de vue de la notion classique de sécurité (rapport de contraintes), de la notion de sécurité ramenée aux causes (rapport des pressions) on de la notion probabilistique.

Nous allons reprendre ces différents points.

\section{2) Sécurité probabilistique.}

Le fait d'essayer chaque tuyau à une pression intérieure correspondant à $\mathrm{C}$ fois la pression de calcul (et pour Kurobe IV nous proposons $C=$ coefficient de sécurité du marché) fait qu'il ne s'agit plus d'un tuyau probable fait de matières probables, mais d'un tuyau certain constitué de matières certaines, puisqu'on réalise le test réel.

Dans cette notion de probabilités composées, tout ce qui se rapporte au produit sortant de l'usine est donc affecté d'une certitude absolue.

II reste, dans le développement de probabilités composées, la notion même de probabilité des causes. Dans notre cas, c'est évidemment la probabilité de surpression qui devient le facteur diterminant.

Or, il ne faul pas oublier qu'une conduite autofrettée est un ouvrage composite, finalement tres hyperstatique el formé d'une multitude d'éléments distincts (frettes) liés à une tôle continue. 


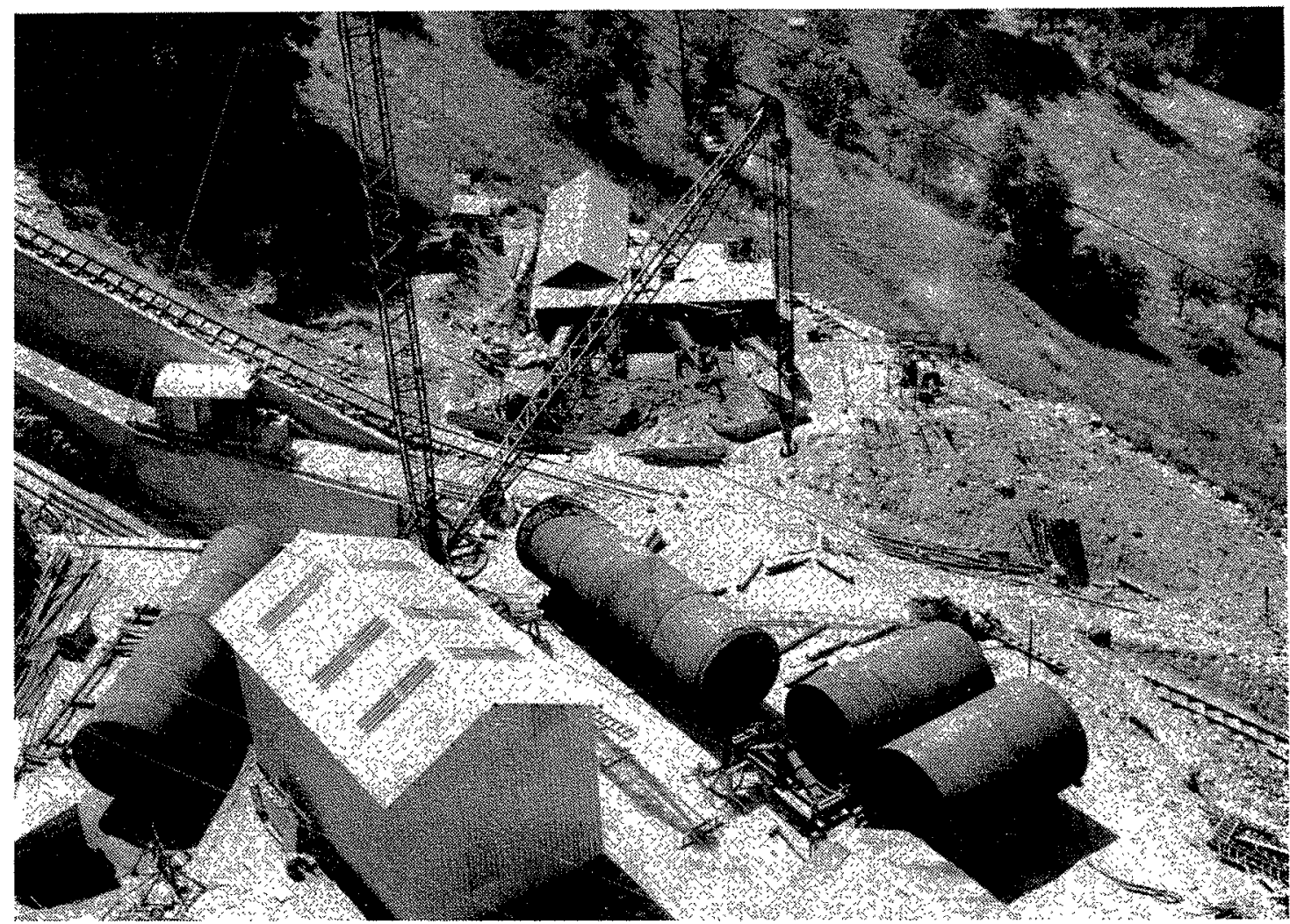

FIG. 3

Photographie montrant les tuyaux auto-frettés à double paroi de la conduite de Roselend (lot $\mathrm{n}^{\circ} 3$ ) de 11 mètres, poids 32 tomes, avant leur descente en galerie. Dans la même galerie, on a descendu des tuyaux surpressés à $2 \%$. et auto-frettés, longueur 11 mètres, J'un poids de 46 tonnes. Les tuyaux arrivaient de Grenoble en longueurs de $5,50 \mathrm{~m}$. Ils ctaient assemblés deux par deux par soudure automatique «Unionmelt» dans un baraquement visible sur la photographic.

Il sufit de regarder les taux de contraintes circonférentielles dans le cas de la conduite de Kurobe IV, dont le calcul complet de la section en amont du coude inférieur est joint, pour relever les chiffres suivants :

- Contrainte circonférentielle dans la tôle sous pression de calcul (hauteur statique + surpression + oscillation) après diminution de $2 \mathrm{~mm}$ de l'épaisseur de la tòle sous l'eftet de Ia corrosion $=+5,4 \mathrm{~kg} / \mathrm{mm}^{2}$.

-... Contrainte circonférentielle dans la frelte dans les mêmes condilions $=+42,6 \mathrm{~kg} / \mathrm{mm}^{2}$.

Ceci veut dire que, pour une cause extrandinaire que l'on ne peut pas soupçonner, - car si on la connaissait on en tiendrait compte, et s'il n'y avait aucune cause d'incertitude, en toute logique, on serait dans le cadre d'un problème déterministe, donc, on ne prendrait pas de coefficient de sécurité; ces chiffres veulent donc dire que si une cause extraordinaire se produisait, le plus grand risque que nous au- rions serait la rupture d'une frette (sur un total de 4570 frettes) et tout nous laisse penser que la conduite ne s'en porterait pas plus mal.

Ceci, bien entendu, dans le cadre d'un choix judicieux des « coefficients de sécurité».

\section{3) Sécurité classique.}

Par procédé de fabrication même, nous amenons le tuyau à une pression de freltage. Dans le cas de Kurobe IV. la pression de fretlage est :

- 2,2 P pour la partie en amont de la première culotte,

- 2,4 P pour le collecteur.

Ceci revient à dire que nous n'offrons pas des coefficients tirés d'opérations algébriques, mais un essai irrefutable.

En poursuivant même ce raisonnement, on pourrait dire que les spécifications des matières et les contròles auxquels nous attachons beaucoup 


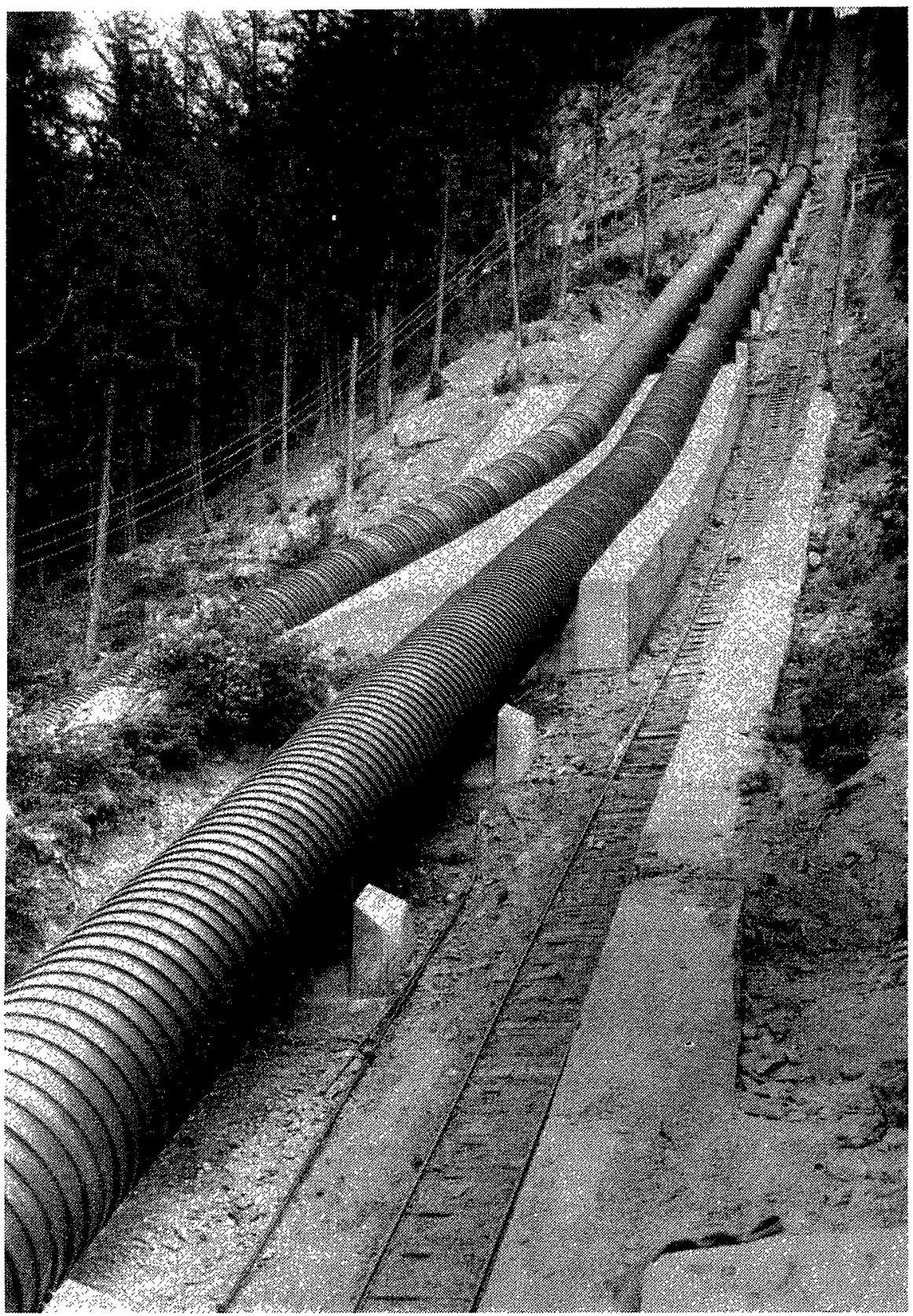

FIG. 4

Conduites forcées

de la chute de Malgovert.

Hauteur de chute : $\quad 750 \mathrm{~m}$ Débit par conduite : $\quad 25 \mathrm{~m}^{3} / \mathrm{s}$ Puissance : $\quad 210000 \mathrm{ch}$ Diamètres : $\quad 2,100 / 2,200 \mathrm{~m}$

(Photo Studio Villeurbannais.) de soins n'ont finalement aucune importance « client » et qu'ils ne servent qu’à garantir à la fabrication la réussite de chaque épreuve, autrement dit, un prix de fabrication de produit livré qui, lui, est forcément conforme à la sécurité requise. C'est évidemment aller un peu loin, mais pourtant cela mérite d'être souligné.

\section{4) Sécurité ramenée aux causes.}

Pour des tuyaux soudés ordinaires (formés d'une tôle roulée), le rapport de la limite élastique de la tôle aux contraintes circonférentielles dues à la pression de calcul est évidemment le même que le rapport des pressions amenant la limite élastique dans la tôle à la pression de calcul. Il en est bien différemment pour des conduiles auto-frettées. Pour faire un exemple, si on prend le cas de la partie hasse de Kurobe IV :

a) Constituée en tôle à $\sigma_{0,2} \ldots \quad 60 \mathrm{~kg} / \mathrm{mma}^{2}$

Epaisseur .............

Diamètre intérieur. . . . . . $3,240 \mathrm{~m}$

Contrainte circonférentielle:

$$
\frac{p \mathrm{R}}{e}=\frac{70,2 \times 1620}{100 \times 42}=27 \mathrm{~kg} / \mathrm{mm}^{2}
$$


Fig. 5

Essai de rupture

d'un tuyau de Kurobe IV.

Caractéristiques :

Diamètre intérieur : $\quad 3164 \mathrm{~mm}$ Hauteur :

Epaisseur :

$18 \mathrm{~mm}$

en CONFOR E $34 / 54$

15 frettes de $100 \times 30$ au pas

de 180: en confon $G$ 95/115

Pression maximale normale de marche :

$63 \mathrm{~kg} / \mathrm{cm}^{2}$

pression de collage : $\quad 42 \mathrm{~kg} / \mathrm{cm}^{2}$

Pression de frettage : $139 \mathrm{~kg} / \mathrm{cm}^{2}$ Pression effective ayant amené

la rupture de 2 frettes $: 210 \mathrm{~kg} / \mathrm{cm}^{2}$ (Photopress, Grenoble.)

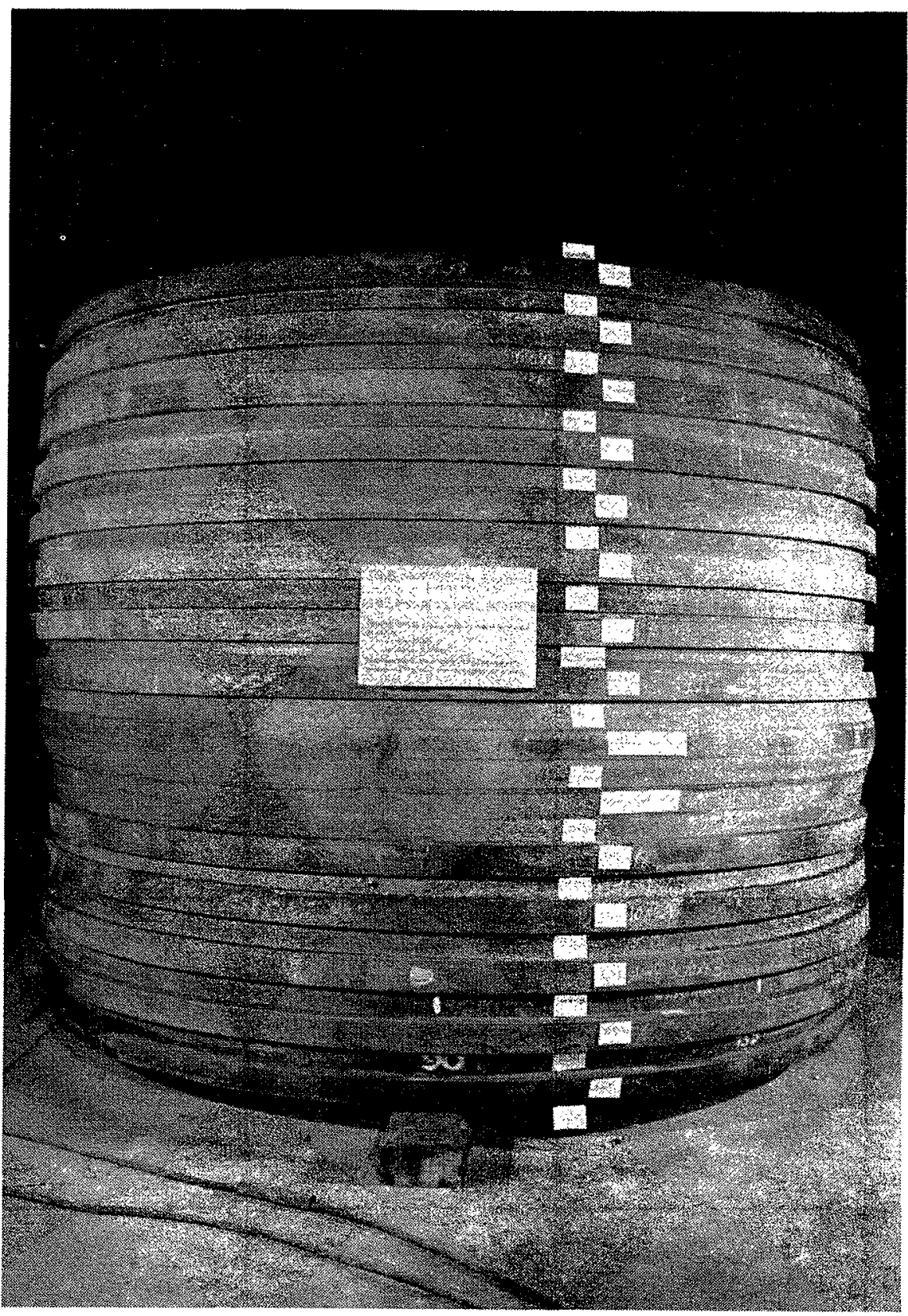

à pression nulle.....

à pression $p \ldots \ldots \ldots$ a frette ...

c'est-à-dire des coefficients de sécurité ramenés aux contraintes $C$.

$$
\begin{aligned}
& \text { Frettes . . C }=\frac{95}{42,6}=2,23 \\
& \text { Tòles. . . C C }=\frac{36}{5,4}=6,65
\end{aligned}
$$

Par contre, le rapport des pressions amenant 


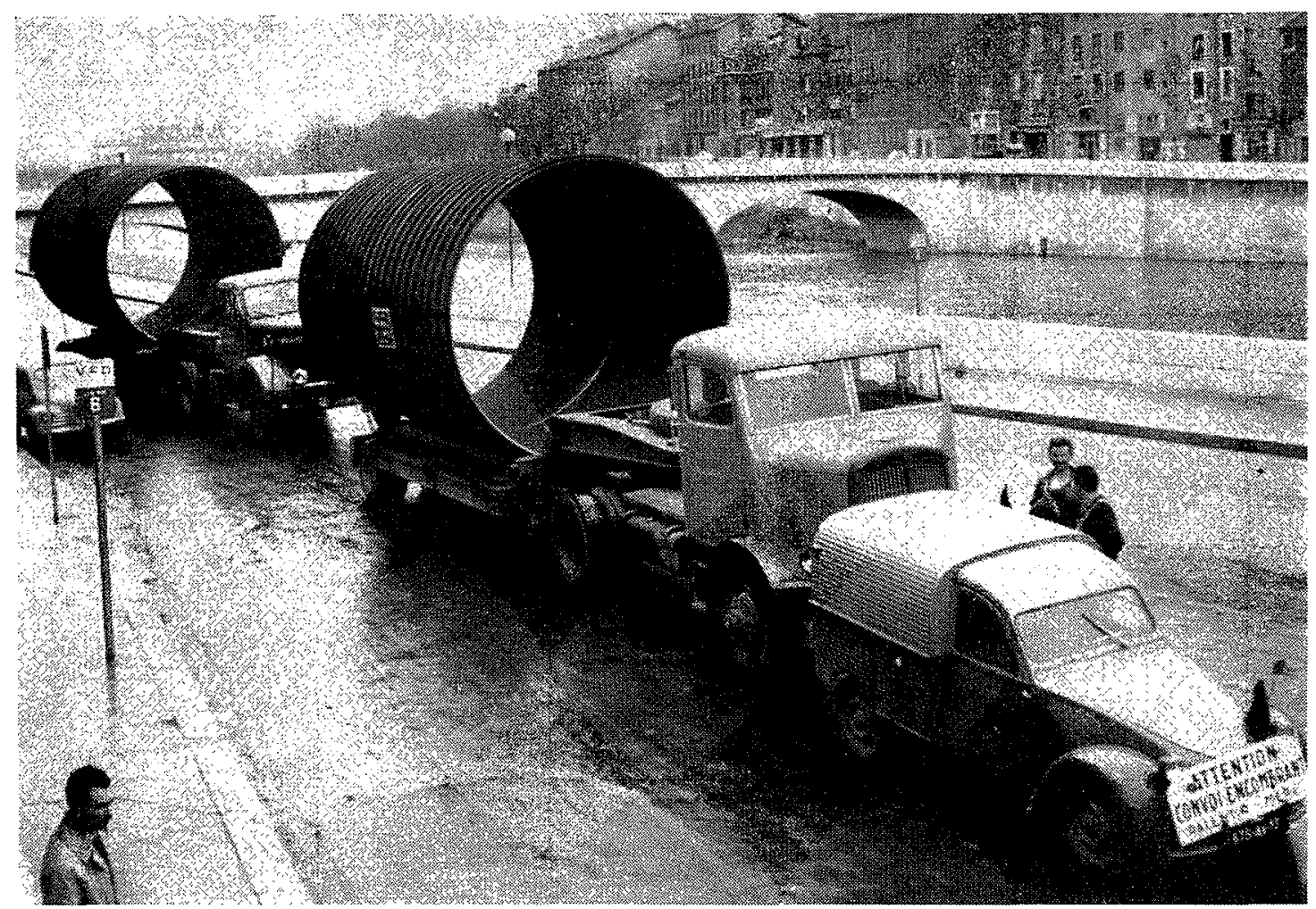

Fig. 6

(Photopress, Gronoblc.)

Tuyaux surpressés à $2 \%$ et auto-frettés de la conduite cie Roselend, en ronte pour le chantier de La Bathie.

la limite élastique dans la conduite est (voir calcul joint) :

$$
\mathrm{K}=2,60
$$

Cette démonstration est indiscutable et la sécurité ramence aux pressions (qui pourrait être définie comme celle de l'exploitant) est bien supérieure à la sécurité ramenće aux contraintes; c'est d'ailleurs le chiffre « sécurité » que nous entendons généralement et c'est celui qui est reproduit dans la liste des quelque 100 références depuis 1949 (notre firme en possède depuis 75 ans) que nous avons envoyées.

Donc, placé à ce point de vue, le choix fait pour Kurobe IV est raisonnablement large, tout en restant raisonnablensent économique, en regard de l'importance de cette installation.

Nous joignons à ce chapitre un croquis montrant en outre les coefficients de sécurité ramenés aux pressions (à titre indicatif bien entendu), et on voit que tous les coefficients pour les différentes sections ramenées aux pressions sont de l'ordre de grandeur de celui que nous venons d'examiner en détail.

Donc, pour nous résumer sur ce point, en dehors des avantages uniques que représente l'épreuve au coefficient de sécurité classique et la structure complexe de milliers de frettes associées à des tubes, sans oublier la forme circulaire de frettes sans soudure. Les conduites auto-frettées ont un coefficient de sécurité, ramené à la pression, nettement supérieur au coefficient de sécurité ramené aux contraintes.

\section{5) Sécurité "généralisée 》.}

Nous arons dit au paragraphe I de cet exposć que, plus on fouille des calculs, plus on définit différents critères, dont l'ensemble forme la sécurité. Par une association d'idées, nous dirons que les ingénieurs se sont longtemps attachés à la valeur d'une contrainte maximale, alors qu'on sait actuellement qu'on ne peut pas ignorer qu'un état de contrainte est un tenseur, et que c'est la vue d'ensemble sur les composantes de ce tenseur de contrainte qu'il faut avoir pour caractériser l'importance d'une sollicitation.

Nous avons indiqué le choix des coefficients de sécurité fondamentaux demandé dans l'appel d'offre, à savoir :

Coefficient de contrainte admissible (par composante circulaire et longitudinale) :

- 2,2 à l'amont de la première culotte, 


\section{- 2,4 au collecteur.}

En fait, nous offrons un complexe de coefficients comprenant évidemment les précédents. A titre indicatif, nous joignons un croquis illustrant les différents coefficients dont l'ordre de grandeur sera conservé ou amélioré, et qui dépendent de dessins précis d'exécution. De toute façon, les coefficients de base indiqués étant les limites inférieures contractuelles.

En nous reportant à l'exemple précis de la section basse dont nous joignons le tableau de calcul, nous rappelons les différents coefficients de sécurité dont l'ensemble forme la « sécurité ».
- Rapport pression essai à pression de calcul ....................... (et ceci est une réalité tangible).

- Coefficient de sécurité des contraintes circonférentielles ramené aux contraintes (limite élastique après corrosion de $2 \mathrm{~mm}$ ) . . . ...............

- Coefficient de sécurité des contrainles circonférencielles ramené aux pressions (limite élastique après corrosion

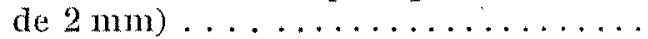

- Coefficient de sécurité des contrainles longitudinales ramenées aux contraintes (limite élastique après corro-
Fric. 7

Vue aérienne de la conduite de Roselend (lot $n^{4} 2$ ) en cours de betonnage, avant yemblaiement.

(Photo R. Bertrand, Alhcrivillo.)

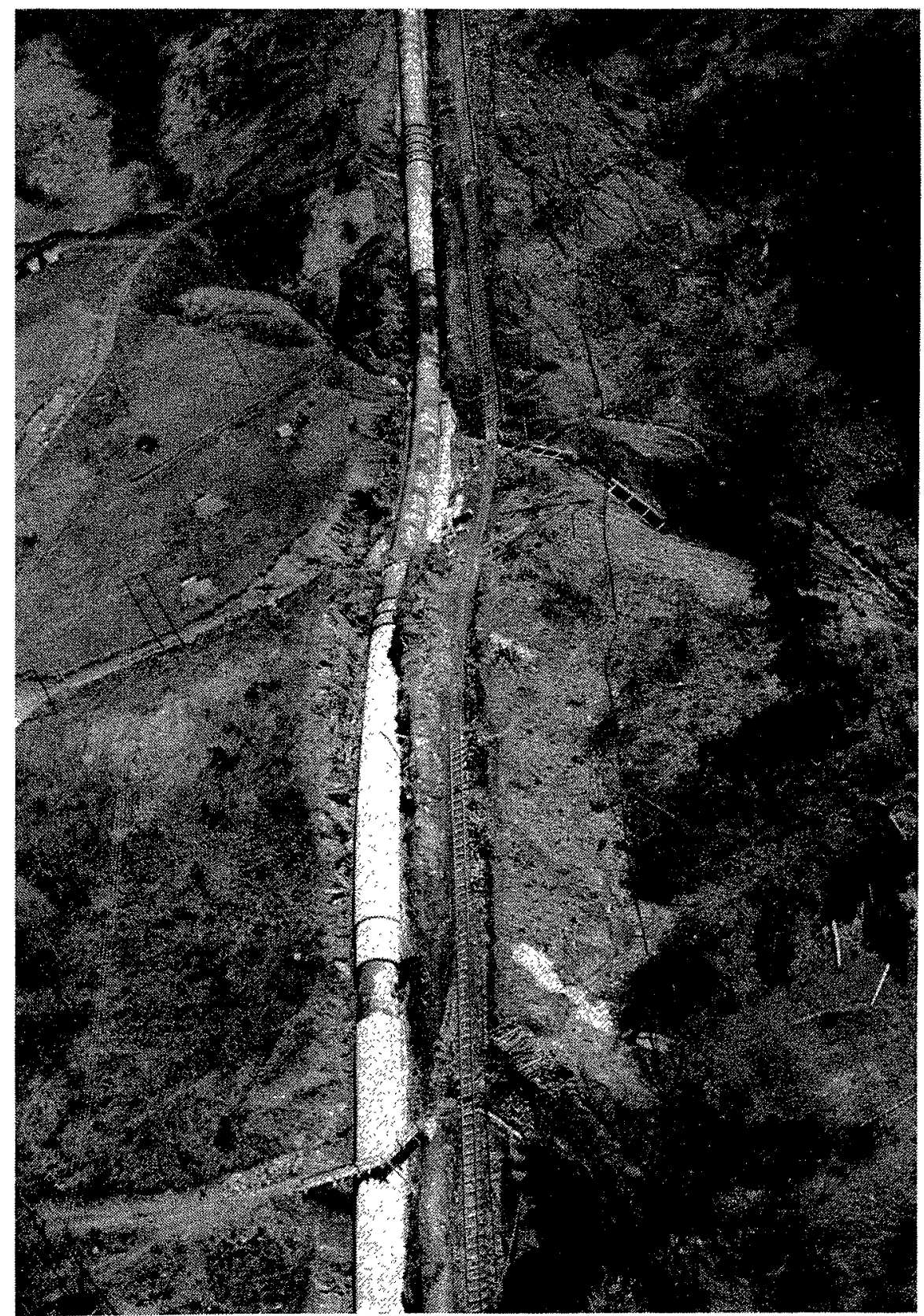




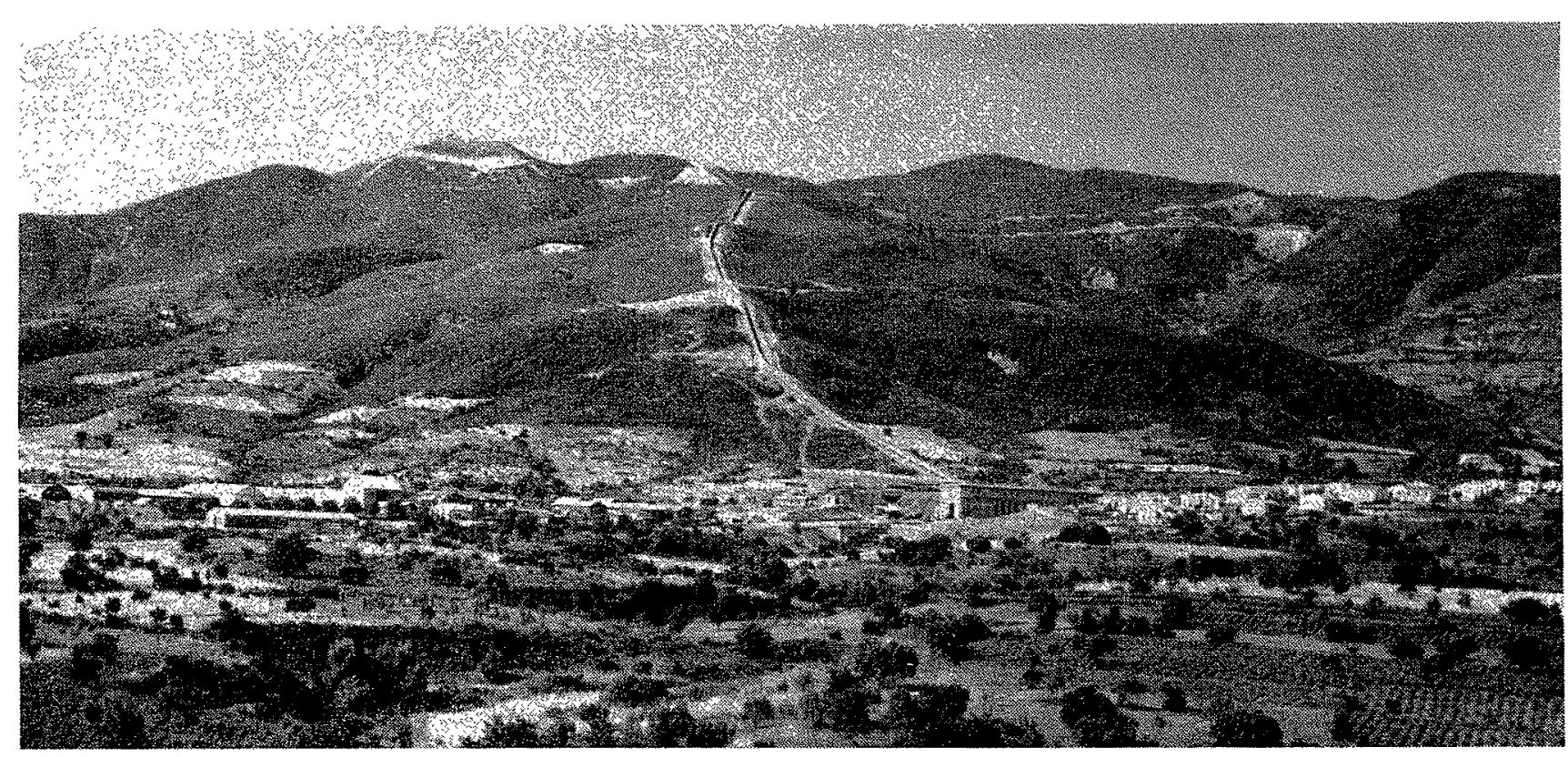

FIG. 8

(Takès 'Arist. Spanias, Karditsa.)

Vue d'ensemble de la conduite forcéc de Megdova (Grèce).

Chute brute :

Débit :

$$
\begin{aligned}
& 585 \mathrm{~m} \\
& 26,4 \cdot \mathrm{m}^{3} / \mathrm{s} \\
& \text { Mise en service : }
\end{aligned}
$$
$\begin{array}{ll}\text { Puissance : } & 170000 \mathrm{ch} \\ \text { Diamétres : } & 2400 / 2600 / 3000 \mathrm{~mm}\end{array}$ juin 1960 sion de $2 \mathrm{~mm}$ au point le plus défavorable, compte tenu de l'efficience de la soudure de chantier de 0,85 )..

- Coefficient de sécurité des contraintes dues au remplissage (limite élastiqune après corrosion de $2 \mathrm{~mm}$ )...

- Coefficient de sécurité an flambage après corrosion de $2 \mathrm{~mm} \ldots . . \ldots \ldots$.

- Coefficient de sécurité de la contrainte équivalente la plus défavorable (limite élastique après corrosion de $2 \mathrm{~mm}$ ). On ne parle que des tôles, les frettes n'étant soumises qu'à des contraintes circulaires............

\section{6) Conclusions.}

«L'Ensemble Sécurité » que nous proposons forme un complexe d'autant plus homogène et sérieux que le produit livré subit l'épreuve en atelier.

Cet ensemble est caraclérisé par les coefficients de sécurité fondamentaux contractuels que nous proposons.

Le choix des valeurs de base est en harmonie avec les références que nous joignons (le coefficient indiqué étant le rapport des pressions contractuelles, le coefficient réel en chaque point étant supérieur).

Mais, encore une fois, du fait de sa structure ef du fait de l'épreuve, il y a encore plus dans une conduite auto-frettée que le tableau, pourtant suffisant en lui-même, des sept composantes de «l'Ensemble Sécurité ».

La valeur $c=2,2$ semble constituer, pour le coefficient contractuel de base, la tendance actuelle lorsqu'il s'agit de conduites forcées de grande puissance et pour autant qu'il s'entende sous pression de marche $P$, soit que, sous $c P$, les contraintes soient au plus égales à $\sigma_{0,2}$, soit que :

$$
c=\frac{\sigma_{0,2}}{\sigma_{p}}
$$

Les deux formules sont identiques lorsqu'il s'agit de TS, mais elles sont quelque peu différentes pour les T.A.F.

$$
* *
$$

Les nouvelles tendances sont aussi faites de plus grandes exigences dans le choix et la réception des matériaux et d'un contrôle plus rigoureux de la construction, notamment dans le domaine de la soudure.

De plus en plus, en effet, on s'oriente vers l'obtention d'une qualité améliorée des matériaux mis en ceuvre, tant en ce qui concerne l'élaboration du métal de base que de sa soudabilité. 


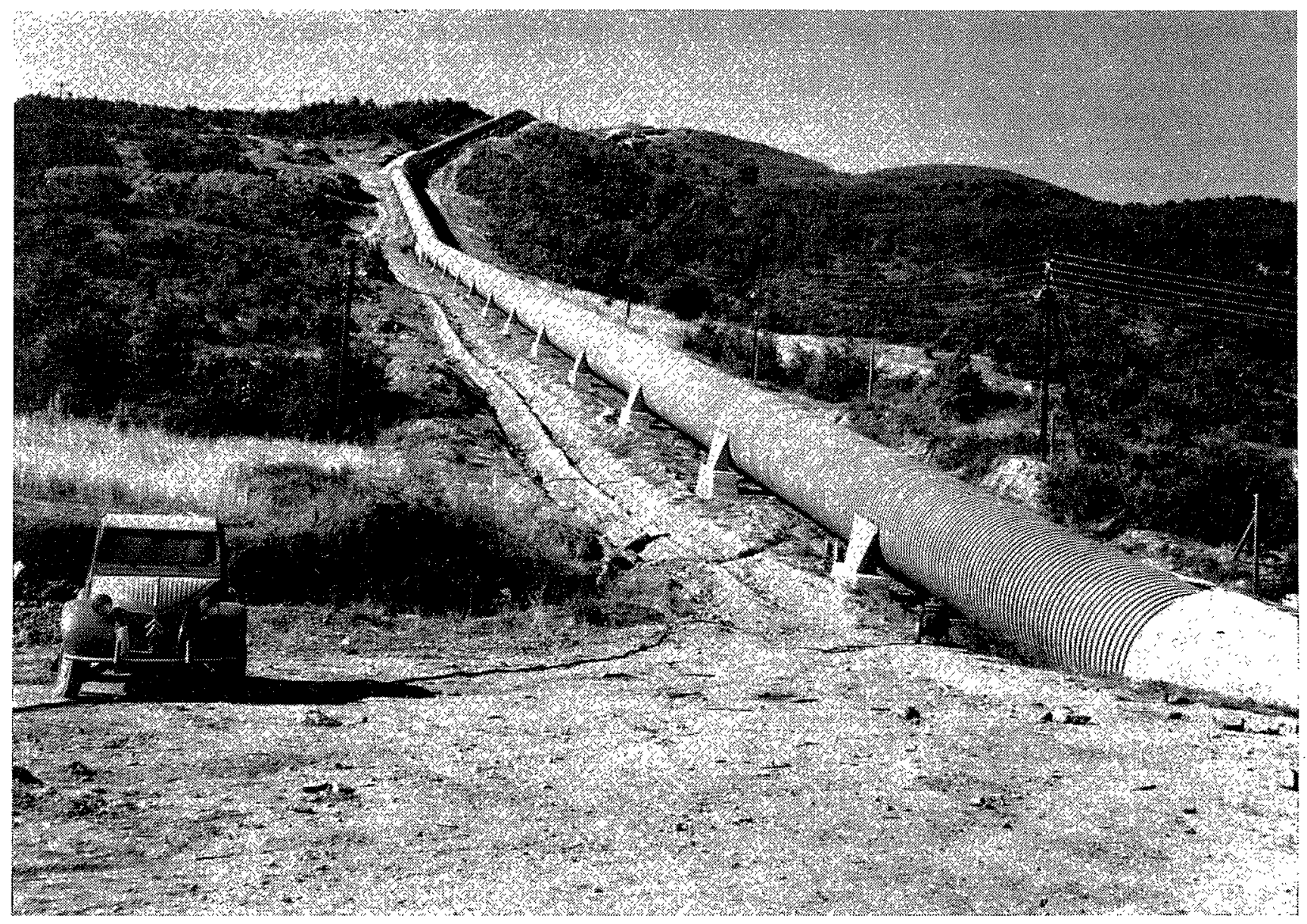

Frg. 9

(Takès 'Arist. Spanitis, Karditsa.)

Vue de la partie inférieure de la conduite de Megdova (Grèce).

Espacement des supports : 16 mètres.

On exige en particulier pour les aciers demidurs $36 / 52$ une garantie de résilience relativement élevée à basse température $\left(4 \mathrm{~kg} / \mathrm{cm}^{2}\right.$ à --20 ou $-30^{\circ} \mathrm{C}$ ), ainsi qu'un rapport relativement élevé $(0,60$ au minimum) de la valeur de la résilience $K_{1}$ à l'ambiante sur métal normalisé soumis à un allongement de $10 \%$ et suivi d'un traitement de vieillissement à $250^{\circ}$, à la valeur de $\mathrm{K}$ de la résilience sur métal normalisé.

Le contrôle ultra-sonique actuellement parfaitement au point, aussi bien sur les soudures de montage et d'atelier que sur le métal de base, permet un contrôle plus rigoureux que les contrôles radiographiques et gammagraphiques pour la recherche des défauts internes, et que ceux par ressuage ou magnétoscopie pour la recherche des défauts proches de la surface. II permet en particulier le contrôle des soudures de montage en galerie blindée, très difficile, sinon impossible, à réaliser en radio ou gammagraphie. Il permet aussi de déceler, comme les dédoublures de tôles, le défaut principal des tuyaux soudés au gaz à l'eau qui équipent en- core de très nombreuses chutes, défaut qui se présente sous la forme d'un collage des bords de la tôle dans la zone de soudure lorsque celle-ci n'est pas réussie. D’autres méthodes de contrôle modernes, tels que la mesure de la microdureté superficielle, l'examen superficiel de structure et l'étude du réseau cristallin par radiocristallographie sont maintenant utilisables dans la pratique courante.

$$
* *
$$

Le cahier des charges de la S.H.F. n'imposait pas de minimum pour la valeur du rapport $E / R$ (E limite élastique à $0,2^{\circ} \%, \mathrm{R}$ résistance à la rupture) des aciers employés.

Actuellement on tend vers $\mathrm{E} / \mathrm{R} \leqslant 0,8$.

Rappelons à ce sujet le point de vue que nous avons souvent défendu : il n'y a pas d'inconvénient à utiliser des aciers où le rapport $E / R$ est grand, toutes les fois que l'on est en présence de pièces qui n'ont pas à subir « l'adaptation » lors 
des épreuves hydrauliques après fabrication ou en service.

C'est d'abord le cas des frettes des tuyaux autofrettés, qui ne subissent que des contraintes tangentielles et dont la forme qui leur est donnée à chaud ne peut différer de la forme théorique que par une légère ovalisation qui disparaît sous l'effet d'une contrainte négligeable par rapport à la contrainte de service. En fait, pour des frettes, les valeurs de $\mathbf{E}$ et de $\mathrm{R}$ peuvent être rapprochées. L'utilisation très large que nous avons faite, avec succès, de frettes souples en fils à très haute résistance, câblés, dont la capacité de déformation est pratiquement nulle, en est la confirmation. A notre avis, il n'y a pas d'inconvénient à admettre pour le rapport $E / R$ la valeur maximale de 0,92 lorsqu'il s'agit de frettes rigides.

C'est ensuite le cas des tuyaux soudés à l'arc électrique qui sont exécutés en partant de tôles planes, mais qui doivent cependant posséder une capacité de déformation non négligeable, du fait que leur forme définitive est obtenue non plus à chaud comme les frettes rigides, mais par cintrage à froid.

Comme pour les frettes, la forme des viroles après transformation des tôles ne diffère de la forme théorique que par une légère ovalisation qui disparait sous l'effet de contraintes qui, sans être négligeables comme pour les frettes - du fait que les tuyaux en service n'ont pas seulement à subir des contraintes tangentielles, mais aussi des contraintes longitudinales - sont cependant faibles en regard des contraintes en service. Il n'y a pas d'inconvénient à admettre pour le rapport $E / R$ la valeur de 0,85 lorsqu'il s'agit de tuyaux soudés. Cette valeur n'est d'ailleurs atteinte pratiquement que sur les tuyaux fortement surpressés ou sur les tuyaux soudés comportant des tôles à très haute limite élastique obtenue par trempe et revenu.

Mais sur les pièces spéciales utilisées, en particulier sur les collecteurs de distribution aux turhines, il en est différemment. Leur forme complexe fait que "l'adaptation 》 inévitable peut souvent donner naissance à des déformations non négligeables et à des contraintes élevées. C'est pourquoi la valeur $E / R$ des aciers utilisés à la construction de ces pièces ne devrait pas être élevée.

La limitation de la valeur $E / R$ à 0,80 , si elle était impérative, éliminerait les frettes en aciers spéciaux de haute qualité aux caractéristiques $105 / 120, E / R=0,875$ ainsi que les tôles trempées donnant $E / R=0,85$. Nous pensons cependant que, tout au moins pour les tuyaux proprement dits, ainsi que pour les frettes, on tolérera 0,85 . Cela permettra l'utilisation des tôles trempées $68 / 80$ déjà admises sur la conduite de Roselend, des frettes en aciers spéciaux 95/115 et des frettes $70 / 100$ surpressées à $2 \%$ donnant $85 / 100, E / R=0,85$ et les tuyaux surpressés jusqu'à $4 \% \mathrm{E} / \mathrm{R}=0,83$.

$$
*
$$

Le cahier des charges SHF prévoyait pour l'épreuve en usine, l'épreuve à $2 \mathrm{P}$ que l'on dénommait « règle d'or *, tant était grande l'importance qu'on y attachait en matière de sécurité d'exploitation.

Maintenant on tend vers une épreuve en usine faite sous une pression déterminant une contrainte ne dépassant pas 80 à $90 \%$ de $\sigma_{0,2}$.

Cette tendance ne rendrait plus impérative l'épreuve à $2 \mathrm{P}$ et priverait le mâtre de l'œuvre de la garantie de sécurité qu'elle lui fournit - en conférant à l'ouvrage un coefficient de sécurité égal à 2 - à moins que les coefficients de sécurité soient pris en conséquence. Pour que l'épreuve à $2 \mathrm{P}$ soit en effet réalisable dans le cadre des dispositions indiquées, il faut :

- pour un tuyau soudé ordinaire :

$$
c=2,5 \text { ou } 2,2 \text { pour } 0,8 \text { on } 0,9 \sigma_{0,2} ;
$$

- pour un tuyau auto-fretté normal :

$$
c=2,33 \text { ou } 2,13 \text { pour } 0,8 \text { ou } 0,9 \sigma_{0,2} ;
$$

- pour un tuyau surpressé ou auto-fretté surpressé :

$$
c \doteq 2
$$

dans tous les cas et par définition.

Cependant, il reste évident - en considération de ce que nous avons écrit au début de cet article - qu'en ce qui concerne les probabilités de rupture, l'avantage des tuyaux surpressés (ordinaire ou auto-frettés) sur les tuyaux soudés ou auto-frettés simples est plus écrasant encore que celui mis en évidence précédemment à l'occasion des commentaires de Kurobe des tuyaux antofrettés sur les tuyaux soudés, et cela toutes les fois que les conduites seront établies sur la base d'un coefficient de sécurité inférieur à ceux que nous venons d'indiquer.

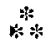

Le maître de l'œuvre a toujours eu le souci de protéger ses installations contre les éléments extérieurs en des zones qu'il sait dangereuses. La tendance actuelle, tout au moins pour les aménagements très importants, est de les protéger d'une façon totale et définitive contre les éléments extérieurs quels qu'ils soient, y compris les sabotages.

Ce problème de protection peut d'ailleurs être traité de façons différentes selon les conditions 
en présence desquelles on se trouve lors de l'étude d'un aménagement déterminé.

Lorsqu'une conduite est établie en roche parfaite, on envisage un aménagement en galerie blindée avec participation optimum du rocher, tout en respectant le critère $c \geqslant I$ (conduite libre). Si l'on n'est pas absolument sûr du rocher, la même solution peut être envisagée, mais avec participation réduite ou nulle; l'aménagement devient très onéreux. On préfère alors installer la conduite libre à l'intérieur d'une galerie ou bien - ce qui à notre avis est mieux encore et plus économique - établir la conduite selon la formule adoptée pour Roselend à l'amont de la partie en galerie blindée. Cette conduite; complètement enterrée, est revêtue sur toute sa longueur d'un important anneau de béton l'enrobant complètement.

On apporte également plus d'attention à la protection des conduites contre la corrosion. On prévoit systématiquement une surépaisseur de corrosion de 1 a $2 \mathrm{~mm}$ selon l'épaisseur et l'on apporte plus de soins aux revêtements intérieur et extérieur.

Pour assurer la stabilité d'une conduite forcée, un volume important de béton, armé ou non, est nécessaire, mais bien souvent des économies assez considérables de béton peuvent être réalisées si l'on s'attache à tenir compte avec précision de tous les efforts absorbés par les massifs d'ancrage. Certes, ceux-ci sont connus depuis longtemps en valeur et en direction, mais certains, jugés de peu d'importance, étaient négligés et le constructeur de conduites forcées chargé de fixer leurs dimensions s'attachait surtout à donner aux massifs d'ancrage des dimensions couvrant très largement la stabilité de la conduite, sans chercher, comme il le fait pour la conduite, à tirer la quintessence de la matière.

Mais il apparânt de plus en plus qu'une étude conduite forcée ne doit plus être menée sous l'angle « poids de la fourniture métallique », mais sous un angle plus ouvert. Le constructenr spécialisé de conduites forcées, que nous avons toujours considéré comme devant être un instal lateur, doit de plus en plus offrir au maitre de l'ouvre la solution qui au total, génie civil compris, se trouve être la plus économique (12).

(12) Travaux, décembre 1957 : «La détermination des efiorts dans les massifs d'ancrage de conduites forcées $»$, par MM. Robert Bovchayen (EBV) et Claude Taschen (SDEM).
C'est ce que le maître de l'ceuvre tend à comprendre de plus en plus dans le cadre de la compétition en ne séparant pas la fourniture de la conduite des travaux de génie civil. C'est la logique même, car l'importance des travaux de génie civil est en liaison directe avec la structure de la conduite. On a d'ailleurs souvent constaté qu'entre deux solutions, ce n'est pas toujours celle ou la conduite est la moins coûteuse qui, en définitive, est la plus avantageuse pour le maître de l'ouvre.

Parmi les tendances acluelles, signalons aussi celle consistant à utiliser des toles soudables à limite élastique très élevée. Comme pour les frettes, la haute limite élastique de ces tôles est obtenue par une trempe suivie d'un revenu à température correspondant à celle nécessaire à l'obtention de la relaxation des tensions internes de soudage. A leur état de livraison, et après le recuit habituel de détente après soudure, ces nuances d'aciers donnent des limites élastiques de l'ordre de $65 \mathrm{~kg} / \mathrm{mm}^{2}$ avec un bon allongement et de bonnes résiliences à l'ambiante et à basse température.

Ces tôles trempées viennent d'être utilisées avec succès en Suisse sur une importante conduite forcée et en France sur la conduite de Roselend pour constituer la paroi externe des tuyaux auto-frettés double paroi de la partie en galerie blindée.

Nous pensons que l'utilisation de ces tôles est particulièrement intéressante à envisager selon la formule de Roselend. En effet, ces tôles sont coûteuses du fait des éléments nobles qu'elles contiennent et, sur le plan économique comme sur celui de la sécurité, elles ne peuvent pas, en tuyaux soudés simples, remplacer les tuyaux auto-frettés. D'autre part, la soudure de ces aciers, notamment an montage, est plus délicate et difficile que celle des tuyaux auto-frettés, dont la paroi est, en général, constituée d'aciers de la nuance $34 / 52$.

En considérant le profil d'une conduite, il existe une zone au-dessus de laquelle l'emploi des tuyaux auto-frettés n'est plus payant, du fait que les frettes que l'on pourrait y mettre sont trop onéreuses ou inconstructibles en raison de leur trop faible section.

Par contre, la continuité peut être assurée avec la formule de Roselend en utilisant des viroles trempées aux lieu et place des frettes. On constitue ainsi des tuyaux auto-frettés à double paroi pour lesquels la question de soudure de montage ne se pose pas. 